\title{
ALGUNAS CONSIDERACIONES SOBRE LA ENFERMEDAD HIPERTENSIVA CRONICA DURANTE EL EMBARAZO
}

\author{
Dr. Eduardo Acosta Bendek*
}

Aproximadamente un $5 \%$ de los embarazos se complican con alguna forma de hipertensión, tales como la enfermedad hipertensiva específica del embarazo o toxemia gravídica, la hipertensión esencial y la hipertensión de tipo renal. Sin embargo, existe un número pequeño de casos que registran hipertensión $(H)$, en los cuales, se manifiesta como un síntoma de un síndrome o de una enfermedad, como sucede en el Síndrome de Cushing, en el Feocromocitoma, en el Hipertiroidismo, etc., en los que una vez hecho el diagnóstico $y$ su tratamiento, se normaliza la presión arterial (P. A.).

Normalmente, durante el embarazo, toda mujer normotensa registra una baja de la $P$. A. en el segundo trimestre de la gestación. En la paciente hipertensa crónica cuando se verifica este fenómeno es signo de un pronóstico favorable para el feto (1). La causa de esta baja tensional en el segundo trimestre del embarazo obedece principalmente al aumento de las sustancias estrogénicas durante este trimestre y a su efecto bloqueador sobre la adrenalina, como lo

* Médico Jefe del Servicio de Maternidad "Leopoldina de Santos" del Hospital de Barranquilla. comprobó Duff (2) en sus experiencias clínicas.

El diagnóstico de $H$. crónica en obstetricia se hace cuando la P. A. alcanza los niveles de $140 \mathrm{Mx}$. y 90 Mn., previo al embarazo, o durante éste si se descubre antes de la 24a semanas de la gestación, o bien, si se mantiene esta hipertensión hasta tres meses después del parto. La hipertensión crónica más frecuente en el embarazo es la de tipo esencial. La de tipo renal se da con menos frecuencia.

\section{MATERIAL}

Con el ánimo de registrar una incidencia más o menos aproximada de hipertensión crónica en el embarazo, se hizo una revisión sobre 1.000 historias clínicas de la Consulta Prenatal del Departamento de Maternidad Leopoldina de Santos del Hospital de Barranquilla. Se tomó el dato de la $P$. A. registrado en la primera visita y se comparó con los registros de consultas posteriores; se obtuvo los resultados siguientes: 17 pacientes clasificadas como hipertensas crónicas que determina una incidencia de $1.7 \%$, relativamente baja comparada con la de autores extranjeros (2); once de estas pacientes tenían menos 
de 24 semanas de gestación y seis más de ese tiempo. Diez pacientes tenían menos de 30 años y siete más de esa edad; se observó mayor frecuencia de pacientes hipertensas en las multíparas. (Cuadro N․ 1).

\section{CUADRO № 1}

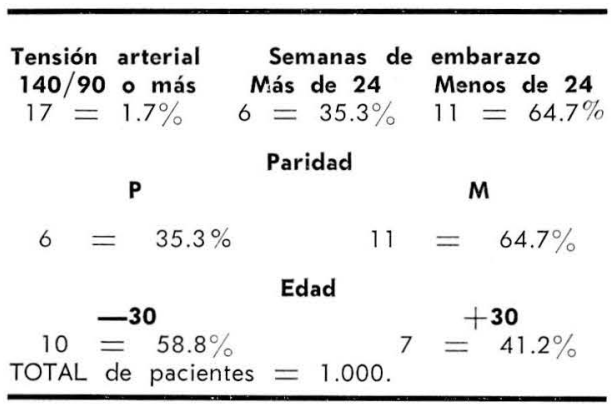

Con el objeto de evaluar las complicaciones y riesgos tanto maternos como fetales en la enfermedad hipertensiva crónica durante el embarazo, se revisó un grupo de historias clínicas de pacientes que habían tenido su parto; se seleccionó 25 de ellas por considerarlas más completas para dicho estudio y se dividieron en cuatro grupos tomando como base para clasificarlas la P. A. diastólica por considerarse de mayor importancia para la paciente embarazada que la P. A. sistólica. (Cuadro № 2).

\section{CUADRO № 2}

\begin{tabular}{rr} 
Grados de Hipertensión & $N^{0}$ de Casos \\
I $90-99$ & $4=16 \%$ \\
II $100-109$ & $8=32 \%$ \\
III $110-119$ & $3=12 \%$ \\
IV 120 y más & $10=40 \%$ \\
TOTAL & $25=100 \%$ \\
\hline
\end{tabular}

Llama la atención que al grupo IV correspondió el $40 \%$ de las pacientes, siguiéndole en su orden el grupo II con un $32 \%$. En los cuadros si- guientes se puede observar el efecto que cada uno de estos grupos ejerció sobre la madre y el feto.

En lo que hace relación a la paridad, se puede observar que la enfermedad hipertensiva crónica es más frecuente en las multíparas; igualmente, se registra un mayor número de hipertensión maligna en este tipo de pacientes, como puede verse en el Cuadro N No 3.

\section{CUADRO N 3}

\begin{tabular}{|c|c|c|c|c|c|c|}
\hline \multirow[t]{2}{*}{ Paridad } & \multirow[t]{2}{*}{$\mathrm{N}^{0}$ de } & \multirow[t]{2}{*}{ casos } & \multicolumn{4}{|c|}{$\begin{array}{l}\text { Grados de } \\
\text { hipertensión }\end{array}$} \\
\hline & & & I & II & III & IV \\
\hline Gran multípara & $3=$ & $12 \%$ & 1 & & & 2 \\
\hline Multípara & $16=$ & $64 \%$ & 2 & 4 & 3 & 7 \\
\hline Primípara & $6=$ & $24 \%$ & 1 & 3 & 1 & 1 \\
\hline
\end{tabular}

Entre las 25 pacientes estudiadas se encontró que 9 de éstas fueron clasificadas como de tipo renal, o sea un $36 \%$, y las 16 restantes, o sea $64 \%$ de tipo esencial. Para esta clasificación se tuvo en cuenta que tanto los exámenes de orina y química sanguínea fuera del embarazo no revelaran signos de insuficiencia renal. Tanto el tipo de hipertensión maligna, como la retinopatía avanzada fue de más frecuente observación en la $H$. de tipo renal. La $H$. esencial registró la mayor incidencia en los mismos casos; cuando no se le sobreagregó la toxemia permitió el desarrollo normal del embarazo con la obtención de un niño sano y a término, lo cual ha sido ya comprobado por varios autores extranjeros (3). De ahí la gran importancia de un buen control prenatal y una estrecha vigilancia en este tipo de pacientes. (Cuadro Ni 4). 
CUADRO № 4

\begin{tabular}{|c|c|c|c|c|c|c|c|c|c|c|c|c|c|}
\hline \multirow[t]{2}{*}{ Tipos de hipertensión } & & \multirow[t]{2}{*}{$N^{\circ}$ de } & \multirow[t]{2}{*}{ Casos } & \multicolumn{2}{|c|}{ Grados de } & \multicolumn{2}{|c|}{ hipertensión } & \multicolumn{2}{|c|}{ Fondo } & \multicolumn{2}{|c|}{ de ojo } & \multicolumn{2}{|c|}{-Grados- } \\
\hline & & & & I & II & III & IV & 0 & I & II & III & IV & V \\
\hline Renal & 9 & $=$ & $36 \%$ & 1 & 1 & & 7 & & 1 & 2 & 1 & & \\
\hline Esencial & 16 & $=$ & $64 \%$ & 3 & 7 & 3 & 3 & & 2 & & & & \\
\hline
\end{tabular}

En el cuadro siguiente puede observarse que la complicación más frecuente en la enfermedad hipertensiva crónica durante el embarazo fue la toxemia con un $60 \%$ de los casos, correspondiendo la frecuencia mayor a la $\mathrm{H}$. de tipo renal, la que se considera como de más serio pro- nóstico feto-materno. Las otras complicaciones encontradas fueron el desprendimiento prematuro de placenta normoinserta y el accidente cerebrovascular. La elevada incidencia de complicaciones en los 25 casos revisados se debió a la ausencia de control prenatal.

CUADRO Nํ 5

\begin{tabular}{lrlccc}
\hline & & & Complicaciones durante el embarazo \\
& & Eclampsismo & Eclampsia & $\begin{array}{c}\text { Desprendimiento } \\
\text { de placenta }\end{array}$ & Hemorragia cerebral \\
Tipos de hipertensión & $9=36 \%$ & 4 & 4 & 2 & 1 \\
\hline Renal & $9=64 \%$ & 5 & 2 & & \\
Esencial & $16=6$ & & & \\
\hline
\end{tabular}

En el Cuadro № 6 se registra que la aparición de los síntomas en estas pacientes, como la proteinuria, el edema, la elevación de la P. A. etc., fue de observación más frecuente en la $\mathrm{H}$. de tipo renal. El $77 \%$ de estas pacientes presentaron complicación de su embarazo antes de la 25a semana, mientras que en el otro tipo de $H$. solo se registró un $31.2 \%$ de complicaciones.

\section{CUADRO NN 6}

Tipos de hipertensión

Aparición de síntomas en semanas

$$
20-24 \quad 21-25 \quad 26-30 \quad 31-35 \quad 36-40
$$

Renal $9=36 \%$

Esencial $\quad 16=64 \%$

En el Cuadro No 7 se aprecia que en la $\mathrm{H}$. de tipo renal, en un 55\% la terminación del embarazo se produjo antes de la 30 . semana y en un $37.5 \%$ en la $\mathrm{H}$. de tipo esencial; des- pués de la 30: semana de gestación se observó una inversión de esta relación así: en el $44.4 \%$ el embarazo se interrumpió en el primer grupo y en el $62.2 \%$ en el segundo grupo. 


\section{CUADRO № 7}

Edad de terminación del embarazo

Tipos de hipertensión

Antes de la 30: semana

Después de la $30^{\text {a }}$ semana

\begin{tabular}{|c|c|c|c|c|c|c|c|c|c|}
\hline Renal & 9 & $=$ & $36 \%$ & 5 & $=$ & $55.5 \%$ & 4 & $=$ & $44.4 \%$ \\
\hline Esencial & 16 & $=$ & $64 \%$ & 6 & $=$ & $37.5 \%$ & 10 & $=$ & $62.5 \%$ \\
\hline
\end{tabular}

En el cuadro siguiente puede verse que en el tipo de $H$. renal, el $33.3 \%$ correspondió a niños inmaduros, el $55.5 \%$ a prematuros y el
$1.1 \%$ a niños de peso normal. En la $H$. de tipo esencial, en el mismo orden anotado, el porcentaje fué el siguiente: $25 \%, 31.2 \%$ y $43.8 \%$.

\section{CUADRO N: 8}

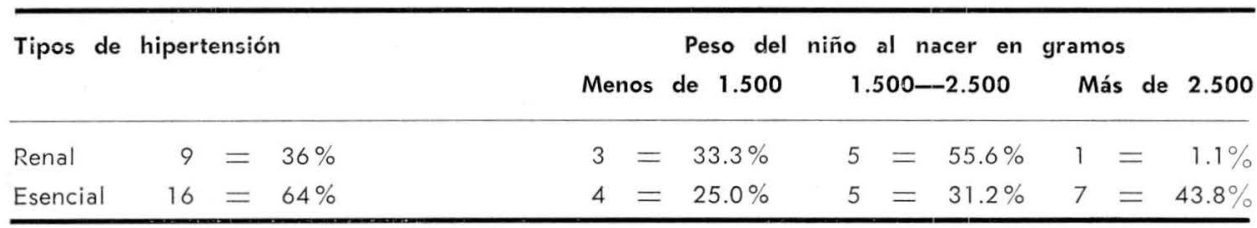

En el Cuadro $N$ ㅇ 9 se observa una mortalidad fetal global que ascendió a un $48 \%$, correspondiendo a la $\mathrm{H}$. de tipo renal la mayor incidencia. La mortalidad materna fue de un $4 \%$ para el grupo de $\mathrm{H}$. de tipo renal en su totalidad.

CUADRO NN 9

\begin{tabular}{|c|c|c|c|c|c|c|c|c|c|c|c|}
\hline \multirow[t]{2}{*}{ Tipos de } & \multicolumn{3}{|c|}{ hipertensión } & \multicolumn{4}{|c|}{$\begin{array}{c}\text { Mortalidad fetal global: } 12=48 \% \\
\text { Grados de hipertensión }\end{array}$} & \multicolumn{4}{|c|}{$\begin{array}{c}\text { Mortalidad materna: } 1=4 \% \\
\text { Grados de hipertensión }\end{array}$} \\
\hline & & & & 1 & II & III & IV & 1 & II & III & IV \\
\hline Renal & 9 & $=$ & $36 \%$ & 1 & & 1 & 5 & & & & 1 \\
\hline Esencial & 16 & $=$ & $64 \%$ & & 3 & 1 & 1 & & & & \\
\hline
\end{tabular}

Por considerarlo de importancia se presenta a continuación el resumen de una historia clínica de una paciente con enfermedad hipertensiva crónica de tipo renal.

D. G. de 28 años; G: III, P: O, Abortos: II; Soltera. Consulta el 21-X-64 por edemas de miembros inferiores, cefálea, sensación de asfixia, P. A. Mx. 220 y Mn. 120. U. R. 26-1II-64. A. U. 24 cms., fetocardia positiva. Embarazo de 26 semanas. Exámenes de laboratorio: Ori- na: proteinuria $300 \mathrm{mg}$. piocitos, eritrocitos y bacterias. Química sanguínea: N. ureico 23.7 mg., urea 50.7 mg., uricemia 6.2 mgs. y creatinina 1.4 mgs. Hematocrito $35 \%$ y 10 grs. de hemoglobina. Diuresis de 900 a 1.000 c.c. en 24 horas. El examen de fondo de ojo fue compatible con una hipertensión angioespástica crónica. Se hizo el diagnóstico de $\mathrm{H}$. de tipo renal maligna. Se sometió a la paciente a reposo absoluto, dieta hiposódica, hipotensores y diuréticos del tipo de las tiazidas. Con este 
tratamiento se mantuvo la P. A. Mx. 200 y 180 y la Mn. 120 y 100. Se usó tres tipos de drogas hipotensoras: la Metil-Dopa, el alcaloide de la Rauwolfia Serpentina y al Dihidrozinoftalazina; cada una de estas drogas se usó por espacio de 4 semanas, no observándose en ninguna de ellas diferencia en lo que se refiere a su efecto hipotensor. La paciente se mantuvo en vigilancia estricta esperando la $36^{\circ}$ semana de su embarazo para la interrupción del mismo. Dos días antes del escogido para la interrupción, inició espontáneamente trabajo de parto. Se obtuvo un niño vivo de $2.200 \mathrm{grs}$. de peso, $45 \mathrm{cms}$. de talla y con un Apgar de 8 . Puerperio normal, manteniéndose la $\mathrm{P}$. A. Mx. 180 y $\mathrm{Mn}$. 110. Durante el estado puerperal se completó el estudio de la exploración funcional renal con la prueba de la sulfofenolftaleína y la urografía excretora, siendo ambas normales. También se le practicó una arteriografía renal usando la vía traslumbar que reveló arterias renales de calibre normal y sistema arterial periférico de la parte media e inferior de am-

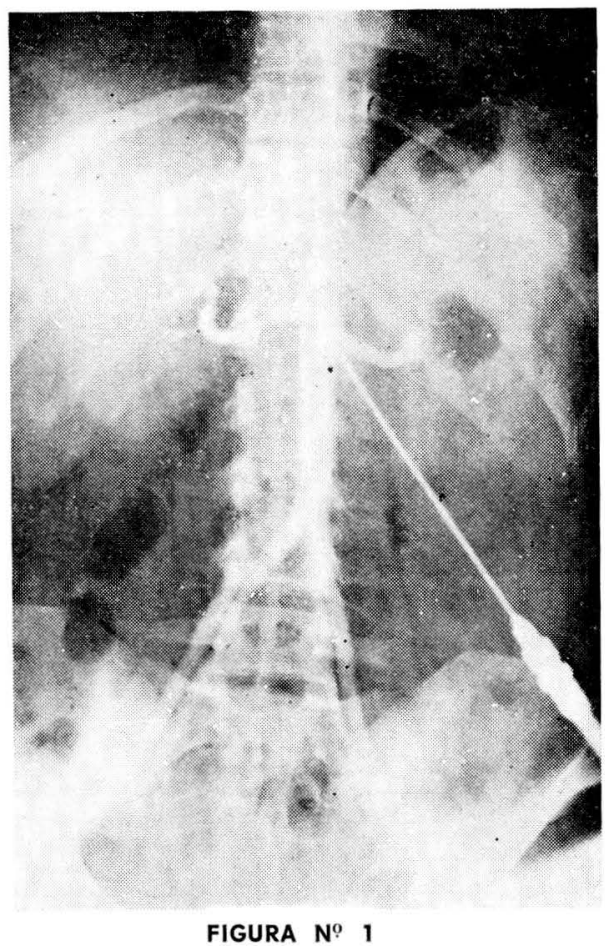

bos riñones también normales. El tercio superior del sistema arterial no se visualizó. En el renograma se puede observar la normalidad del riñón derecho, al paso que el izquierdo no se visualizó bien. Figura No 1 .

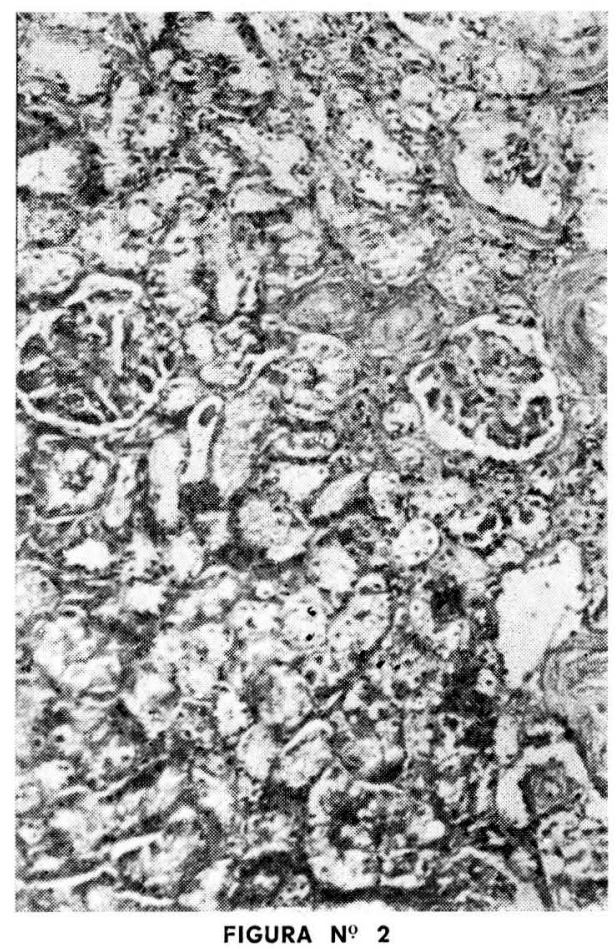

Se completó el estudio renal con una biopsia a cielo abierto, con el siguiente resultado histopatológico: "El examen microscópico revela cambios histopatológicos renales compatibles con una hipertensión. Se aprecia marcado engrosamiento de la membrana basal y proliferación del ovillo glomerular, observándose en algunas áreas, contacto entre el ovillo y la cápsula con reducción del espacio libre. Un glomérulo se presenta totalmente hialinizado. Algunos túbulos muestran un epitelio degenerado y con cilindros. Existe marcado engrosamiento de la pared de los vasos sanguíneos con disminución de la luz de éstos. Se encuentran extravasados un pequeño número de linfocitos y escasos leucocitos polimorfonucleares. No hay evidencia de cambios malignos. 
Los resultados anteriormente analizados muestran que los test de función renal tienen poco valor para indicar la integridad anatómica y funcional del riñón y que sólo el arteriograma y la biopsia renal son los únicos exámenes que revelan el verdadero "status renal" y que permiten obtener una certeza diagnóstica y un pronóstico dentro de cada síndrome vásculo-renal (4).

\section{COMENTARIOS}

Aunque el reducido número de casos estudiados puede considerarse de escaso valor estadístico, permiten, sin embargo, hacer algunas consideraciones sobre la enfermedad hipertensiva crónica durante el embarazo. Se observa así, que la $\mathrm{H}$. de tipo esencial es la más frecuente en el embarazo, la de mejor pronóstico para la madre y el feto, siempre y cuando no se le sobre agregue la toxemia o se encuentre asociada a enfermedad renal (3). De aquí la importancia de un estricto control prenatal que incluya una dieta hiposódica, reposo físico y síquico y tratamiento antihipertensivo bien controlado.

La $H$. cle tipo renal es la más grave $y$ de pronóstico más serio tanto para la madre como para el feto, pronóstico que se hace más sombrío cuando se complica con la toxemia. (5).

La morbimortalidad materno-fetal es mayor en este tipo de hipertensión. A medida que el grado de hipertensión aumenta, el riesgo para el feto y la madre es mayor (6).

En la enfermedad hipertensiva crónica cualquier elevación de la $P$. A. que se presente en el comienzo del embarazo es de pronóstico desfavorable, tanto para la madre como para el feto (7).
En reiación a la conducta que debe seguirse en estas pacientes, se advierte que debe ser muy rigurosa. Toda gestante que en su primera visita registre una $\mathrm{P}$. A. de $140 \mathrm{Mx}$. y de 90 Mn., debe ser chequeada bajo condiciones de reposo en cama por un mínimo de 24 horas con el fin de aclarar su estado de hipertensión. El propósito es prevenir en toda paciente hipertensa embarazada el desarrollo de una toxemia, ya que más del $51 \%$ de los casos que hacen esta complicación en el último trimestre del embarazo producen como secuencia directa una doble mortalidad fetal. Toda paciente hipertensa embarazada que presente una $P$. A. con cifras superiores a 140/90 debe ser hospitalizada a las 35 semanas del embarazo y mantenérsele en una estricta vigilancia, con control de su diuresis, P. A., dieta hiposódica, hipocalórica e hiperproteica, hipotensores, diuréticos y reposo físico y síquico, con el fin de conseguir llegar a las 37 o 38 semanas de embarazo y proceder a la interrupción. Cuando el eclampsismo se sobreagrega antes de la 34. semana, esta interrupción debe hacerse a las 35 o 36 semanas, ya que el riesgo fetal aumenta y más, si se trata de una enfermedad hipertensiva de tipo renal. La $H$. esencial que no esté asociada a lesión renal o vascular, no se agrava con el embarazo $(8,9)$. Toda paciente embarazada con enfermedad hipertensiva tipo renal debe ser internada a las 32 a semanas de embarazo e interrumpirse éste una vez cumplidas las 36 semanas de gestación, ya que el riesgo de muerte fetal aumenta pasada esta fecha (3). En este tipo de pacientes el embarazo aumenta la lesión renal y cada embarazo la agrava más, por lo cual se aconseja en tales casos evitar nuevos embarazos. 
El uso de los hipotensores ha demostrado hasta la fecha ser de poco valor en el tratamiento de estas pacientes. Su manejo inadecuado puede producir muerte fetal, al disminuir la circulación feto-materna (10); lo cierto es, que el pronóstico fetal no mejora con el tratamiento de las drogas hipotensoras.

Sin embargo, no deja de tener importancia la selección de la droga hipotensora en este tipo de pacientes, siendo la más recomendable la de acción central, tipo alcaloide de la rauwolfia serpentina, veratrum viride, derivados ftalazínicos, etc. Con relación a los alcaloides de la rauwolfia serpentina, últimamente se ha considerado la posibilidad de que esa droga produzca una deplesión de las reservas de catecolaminas en el feto, en el recién nacido y en la madre que ha sido sometida a tratamiento. La importancia de lo anterior reside en que algunos investigadores (11) le dan a las catecolaminas un gran papel en la termogénesis del recién nacido.

\section{RESUMEN Y CONCLUSIONES}

1. Se hizo una revisión de mil (1.000) historias clínicas de la consulta prenatal y se encontró una incidencia de hipertensión de $1.7 \%$.

2. Las pacientes hipertensas se subdividieron en cuatro grados de acuerdo a la P. A. mínima, correspondiendo al grado IV el mayor número.

3. La multiparidad resultó ser el grupo más afectado.
4. La hipertensión esencial resultó ser la más frecuente, la de complicaciones menos serias y de mejor pronóstico cuando se somete la paciente a un buen control prenatal.

5. La toxemia es la complicación sobreagregada más frecuente en estas pacientes: es de fácil prevención, pero agrava el pronóstico fetal.

6. La enfermedad renal es de pronóstico más serio tanto para la madre como para el feto.

7. Hasta el momento el tratamiento con las drogas hipotensoras no ha mejorado la supervivencia fetal.

\section{BIBLIOGRAFIA}

1 BROWNE J. C. y cols.: J. Obst. Gynaec. Brit. Emp. 60: 141, 1953.

2 DUFF R. S.: A Symposium on Non-Toxaemic Hypertension in Pregnancy. Little, Brown and Co. Boston. 1958

3 TENNEY B., DANDROW R. V.: Am. J. Obst. \& Gynec. 80: 8, 1961.

4 GUILHEM P., PONTONNIER G., SUE J. M., Progresos de Obstetricia y Ginecología. 6: 234,1963

5 JOHNSON G. T.: A Symposium on NonToxaemic Hypertension in Pregnancy. Little, Brown and Co. Boston, 1958.

6 MARTINI J. L.: Memorias IV Congr. Méx. Gin. Obst. 2: 227, 1963.

7 BROWNE F. J.: Brit. Med. J. 1: 851, 1957.

8 CHESLEY L. C. y cols.: Am. J. Obst. \& Gynec. 53: 851, 1947

9 REID D. E., TEEL H. M.: Am. J. Obst. \& Gynec. 35: 305, 1938.

10 MENDELSON C. L.: Cardiac Disease in Pregnancy. F. A. Davis Company. Philadelphia. 1960

11 TOWELL M. E. y cols.: Am. J. Obst. \& Gynec. 92: 711, 1965. 